\title{
Overview of the CLAS/JLAB Physics Program
}

\author{
S. Stepanyan \\ Jefferson Lab
}

\begin{abstract}
.
The CLAS collaboration has developed a broad program for studying electromagnetically induced processes with both electron and photon beams. This program includes topics such as excited baryon resonances, meson production, hadron structure, search for pentaquarks, and the structure of nuclei. In this report, highlights of recent CLAS results will be presented.
\end{abstract}

Keywords: CLAS, hyperon, pentaquark, DVCS, correlations

PACS: 13.60.-r, 13.60.Fz, 13.60.Rj, 14.20.Jn, 24.85.+p,25.30.Rw

\section{INTRODUCTION}

The experimental program at Jefferson Lab using the CLAS detector covers a wide range of topics of hadronic physics. Studying the spectrum of the baryon resonances and the transition form factors are one of the central issues. Electroexitation of nucleon resonances, as an effective tool in studying the nucleon structure in the regime of strong QCD and confinement, has been the major part of the CLAS physics program. A large effort is devoted to the baryon and meson spectroscopy. Experiments with nuclear targets provided important information on the short range nucleon correlations in nuclei. Dedicated high statistics experiments put stringent limits on the exotic pentaquark production. In this report, highlights of recent CLAS results will be presented.

\section{CLAS DETECTOR AT JEFFERSON LAB}

The CLAS detector at Jefferson Lab is a multipurpose, large acceptance spectrometer consisting of Drift Chambers, Scintillator Counters, gas threshold Cherenkov Counters, and Electromagnetic Calorimeters [1]. Tracking and identification of stable charged particles are performed in the laboratory polar angular range of $8^{\circ}$ to $140^{\circ}$. The PID system allows $\pi / K$ separation for up to $2 \mathrm{GeV} / \mathrm{c}$ and $\pi / p$ for up to $3 \mathrm{GeV} / \mathrm{c}$. For detection and identification of neutrons and photons electromagnetic calorimeters in the forward region are used.

CLAS runs experiments with electron and photon beams, using a variety of solid and liquid targets, as well as longitudinally polarized targets. In electron experiments instantaneous luminosity of about $2 \times 10^{34} \mathrm{~cm}^{-2} \mathrm{~s}^{-1}$ have been achieved. In photon runs, maximum luminosity is limited by accidentals in the tagging system and the acceptable photon flux is about $5 \times 10^{7}$ photons per second. 

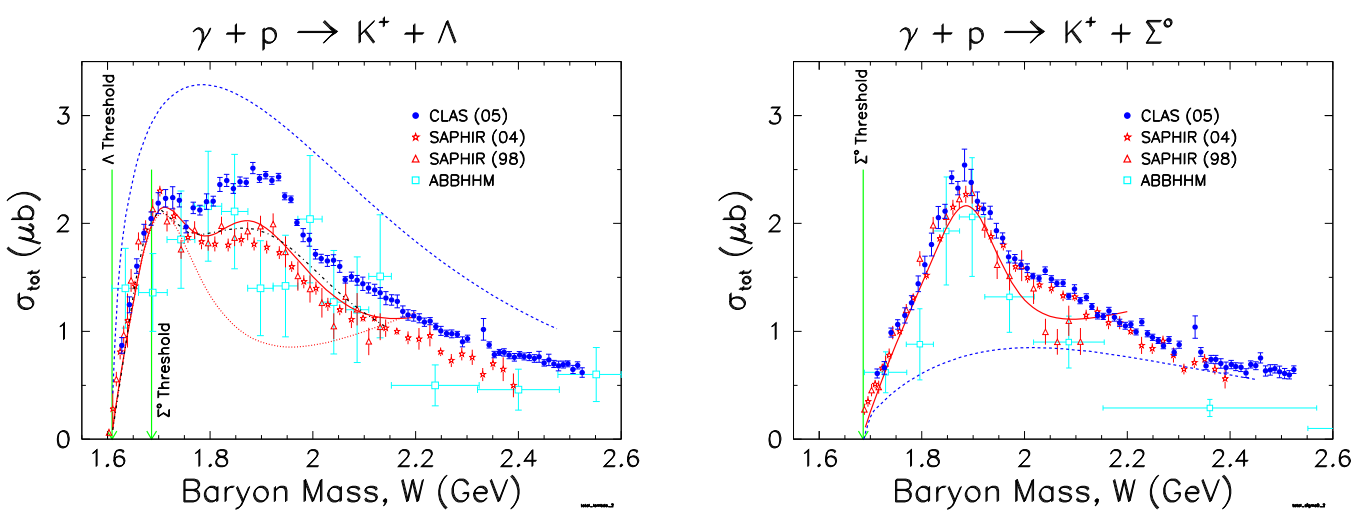

FIGURE 1. Total cross sections for $\gamma p \rightarrow K^{+} \Lambda$ (left) and $\gamma p \rightarrow K^{+} \Sigma^{0}$ (right) reactions from CLAS data (blue circles). Red stars and triangles are data from SAPHIR. The curves are from Regge model (dashed line), KAON-MAID (solid red), KAON-MAID with $D_{13}(1895)$, and calculations by Saghai et al. (dot-dashed black).

\section{$N^{*}$ PHYSICS}

A large set of data in a single- and multi-pion and $\eta$ production reactions have been collected and together with state-of-the-art analysis techniques produced several important results. The $N \Delta$ transition mutipoles at small and large photon virtualities revealed the importance of the nucleon pion cloud [2, 3, 4]. Studies of Roper photocoupling amplitudes showed strong $Q^{2}$ dependence, best, described in terms of large meson cloud contribution. Searches for a new baryon states in two-pion photo- and electroproduction reactions suggested a need for a new state near $1.72 \mathrm{GeV}$ with $J^{P}=3 / 2^{+}$.

\section{Photoproduction of $\Lambda$ and $\Sigma^{0}$ hyperons}

There are many baryon resonances above the strangeness production threshold, predicted by quark models [5], that are not established yet. The hyperon production can be used to search for resonances previously "hidden" from view.

CLAS photoproduction data on $\Lambda$ and $\Sigma^{0}$ hyperons are the largest data set for these channels to-date [6]. The amplitude analysis of differential cross sections was performed using 1377 data points in $K^{+} \Lambda$ and 1280 data points in $K^{+} \Sigma^{0}$ channels. In Fig. $1 \mathrm{~W}$ dependence of the total cross sections for $\gamma p \rightarrow K^{+} \Lambda$ and $\gamma p \rightarrow K^{+} \Sigma^{0}$ reactions are shown. CLAS data, shown in blue circles, show prominent peak near $1.9 \mathrm{GeV}$ for $K^{+} \Lambda$ case and overshoot Bonn results (red symbols). In the case of $K^{+} \Sigma^{0}, \sigma_{t o t}$ shows the previously seen peak at $1.88 \mathrm{GeV}$ and agrees with Bonn results. Curves on the figure are model calculations. It should be noted that models were tuned to describe previous data.

The forward peaking of the $K^{+} \Lambda$ cross section suggests that there is substantial contribution to the reaction mechanism from $t$-channel processes, even in the nucleon resonance region. A phenomenological scaling of the $t$ dependence of the cross sections by $s^{2}$ describes the $K^{+} \Lambda$ data, while it fails to work for the $K^{+} \Sigma^{0}$ case. 

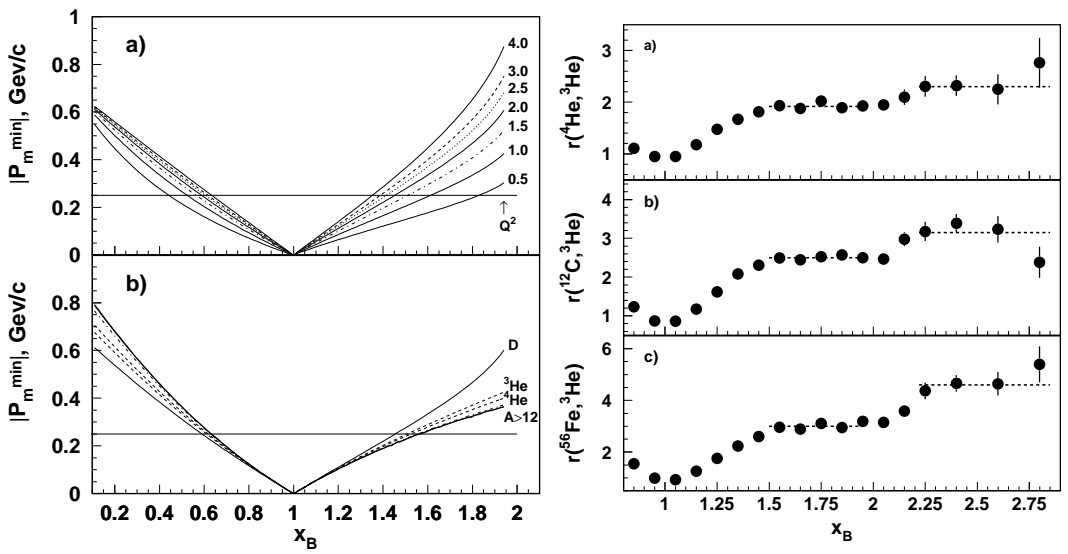

FIGURE 2. Left graph: The minimum recoil momentum as a function of $x_{B}$. (a) For deuterium and (b) for different nuclei at $Q^{2}=2(\mathrm{GeV} / \mathrm{c})^{2}$. Right graph: weighted cross section ratios of (a) ${ }^{4} \mathrm{He}$, (b) ${ }^{12} \mathrm{C}$ and (c) ${ }^{56} \mathrm{Fe}$ to ${ }^{3} \mathrm{He}$ as a function of $x_{B}$ for $Q^{2}>1.4 \mathrm{GeV}^{2}$. The horizontal dashed lines indicate the $N N$ and $3 N$ scaling regions used to calculate the per-nucleon probabilities for 2- and $3 N$ SRCs in nucleus $A$ relative to ${ }^{3} \mathrm{He}$.

\section{NN SHORT RANGE CORRELATIONS}

Understanding the short-range correlations (SRC) in nuclei has been one of the persistent though rather elusive goals of nuclear physics for decades. The SRC, produced by the $N N$ interaction at distances less than the average inter-nucleon distance, result in the universal shape of the nuclear wave function for all nuclei at $p>p_{F}$ (see, e.g., Refs. [7, 8, 9]). For quasi-elastic scattering, $A\left(e, e^{\prime}\right), x_{B}, Q^{2}$, and the minimum recoil momentum of the $A-1$ system are related through energy and momentum conservation. For any nucleus $A$ and fixed $Q^{2}$, there is the value of $x_{B}^{0}$ such that at $x_{B}>x_{B}^{0}$ the minimum recoil momentum contributing to the reaction exceeds the Fermi momentum, $p_{m}>p_{F}$, as shown in Fig.2. Therefore the cross section ratios for heavy and light nuclei should be independent of $x_{B}$ and $Q^{2}$ in the high recoil momentum region.

CLAS electroproduction data on ${ }^{3} \mathrm{He},{ }^{4} \mathrm{He},{ }^{12} \mathrm{C}$, and ${ }^{56} \mathrm{Fe}$ targets were used to show the scaling behavior in $2 N$ and $3 N$ SRCs regions and to obtain the probability for $\mathrm{NN}$ $\mathrm{SRC}$ in different nuclei $[10,11]$. In Fig. $2 x_{B}$ dependence of the weighted cross section ratios of ${ }^{4} \mathrm{He},{ }^{12} \mathrm{C}$, and ${ }^{56} \mathrm{Fe}$ to ${ }^{3} \mathrm{He}$ for $Q^{2}>1.4 \mathrm{GeV}^{2}$ are presented. Two regions of the scaling, $1.5<x_{B}<2$ and $x_{B}>2.25$, shown by dashed lines, correspond to regions of dominance of $N N$ and $3 N$ SRCs in the nuclear wave function, respectively. The absolute probabilities of $2 N$ and $3 N$ SRCs were obtained by integration the momentum distributions in deuterium $(p>0.275 \mathrm{GeV} / \mathrm{c})[10,12]$ and ${ }^{3} \mathrm{He}(p>0.5 \mathrm{GeV} / \mathrm{c})[13]$. The obtained results for absolute probabilities are presented in Table 1. Nuclei with $A<56$ in $99 \%$ of the time compose of a single particle and 2 - and $3 \mathrm{~N}$ correlations. 
TABLE 1. Absolute probabilities of a single particle state, two nucleon and three nucleon correlations in different nuclei. Results for ${ }^{2} \mathrm{H}$ are from integration of the momentum distribution in deuterium for $p>0.275 \mathrm{GeV} / \mathrm{c}$.

\begin{tabular}{cccc}
\hline Nuclei & Single Particle (\%) & $2 N$ SRC $(\%)$ & $3 N$ SRC $(\%)$ \\
\hline${ }^{56} \mathrm{Fe}$ & $76 \pm 4.7$ & $23 \pm 4.7$ & $0.79 \pm 0.25$ \\
${ }^{12} \mathrm{C}$ & $80 \pm 4.1$ & $19.3 \pm 4.1$ & $0.55 \pm 0.18$ \\
${ }^{4} \mathrm{He}$ & $86 \pm 3.3$ & $19.3 \pm 3.3$ & $0.42 \pm 0.14$ \\
${ }^{3} \mathrm{He}$ & $92 \pm 1.6$ & $8 \pm 1.6$ & $0.18 \pm 0.06$ \\
${ }^{2} \mathrm{H}$ & $96 \pm 0.7$ & $4 \pm 0.7$ & - \\
\hline
\end{tabular}

\section{DEEPLY EXCLUSIVE PROCESSES}

Generalized Parton Distributions (GPDs) $[14,15,16]$ became a standard tool for studying nucleon structure. The GPDs contain information on quark/antiquark correlations, and on their transverse and angular momentum distributions. At twist-two level there are four GPDs, denoted $H, \tilde{H}, E$, and $\tilde{E}$. CLAS developed a broad program for studying GPDs using Deeply Virtual Meson Production and Deeply Virtual Compton Scattering (DVCS).

DVCS is the cleanest reaction to study GPDs. Experimentally, the simplest observables to measure are single spin asymmetries where only the imaginary part of the DVCS amplitude and the DVCS and BH interference term survive. At JLAB kinematics the BH dominates in the total cross section. Thus, the $\phi$ modulation of the beam and target spin asymmetries are determined by the imaginary part of the interference term. In JLAB kinematics the $\sin \phi$ moment of $A_{L U}$ is dominated by GPD $H$. The $\sin \phi$ moment of $A_{U L}$ is dominated by $H$ and $\widetilde{H}$, while contributions from $E$ and $\widetilde{E}$ are kinematically suppressed.

In Fig. 3 new CLAS results on the $Q^{2}$ and $t$-dependences of $\sin \phi$ moment of $A_{L U}$ are presented. The data are from the analysis of CLAS data at $4.8 \mathrm{GeV}$ using the technique introduced in [17]. The experimental data are best described by the GPD based model calculations when target mass corrections are included and the skewedness parameter for sea quarks is $b_{\text {sea }}=2$ [18].

Recently, CLAS published the first results on $A_{U L}$ using electron scattering data on longitudinally polarized ${ }^{15} \mathrm{NH}_{3}$ target at $5.7 \mathrm{GeV}$ [20]. In this analysis the contribution of $\pi^{0}$ background was subtracted from $A_{U L}$. In Fig.4 $t$ and $\xi$ dependences of the $\sin \phi$

moment of $A_{U L}$ are presented. A clear $\xi$-dependence of $A_{\mathrm{UL}}^{\sin \phi}$ is seen, with asymmetries increasing with $\xi$. The measured asymmetry is consistent with predictions of a large contribution from GPD $\widetilde{H}$.

\section{SEARCH FOR PENTAQUARKS}

Dedicated high statistics photoproduction experiments on deuterium and hydrogen did not find evidence for $\Theta^{+}$pentaquark. New results were in direct contradiction with 

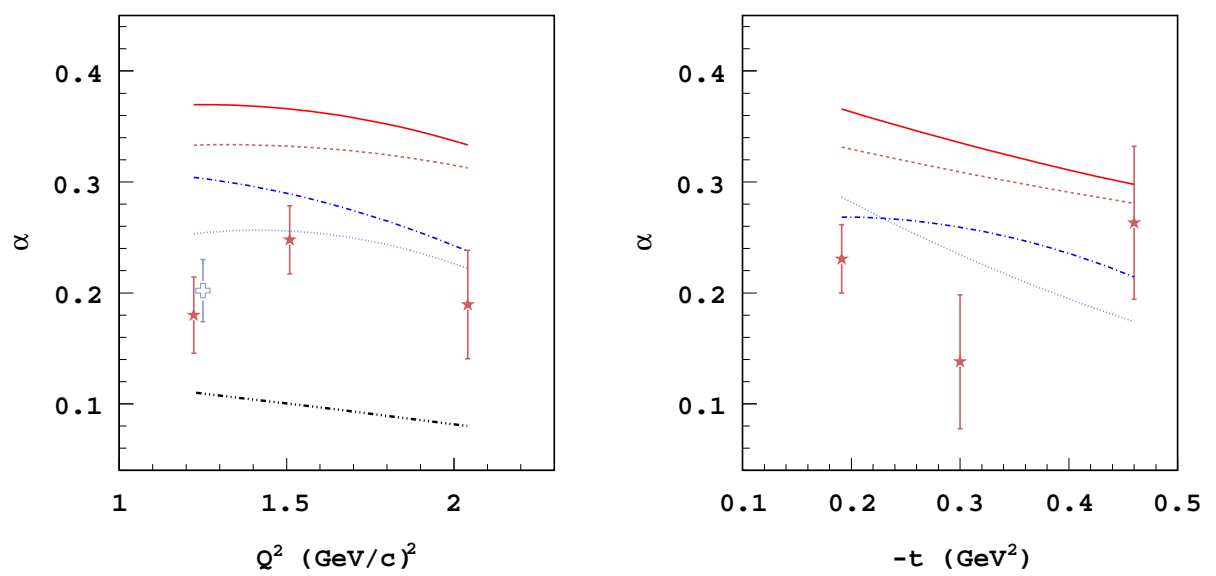

FIGURE 3. The $Q^{2}$ (on the left) and $t$ (on the right) dependence of DVCS asymmetries. The "cross" symbol is the asymmetry measured by CLAS with $4.2 \mathrm{GeV}$ data. The solid and dashed lines are the GPD based model [18] calculations without target mass correction and the skewedness parameter for sea $b_{\text {sea }}=1$ and $b_{\text {sea }}=2$, respectively. The dotted and dotted-dashed lines are calculations with target mass correction and with $b_{\text {sea }}=1$ and $b_{\text {sea }}=2$, respectively. The dashed-dot-dot-dot line is the result of calculations using Regge model for interaction of the photon with the proton [19].

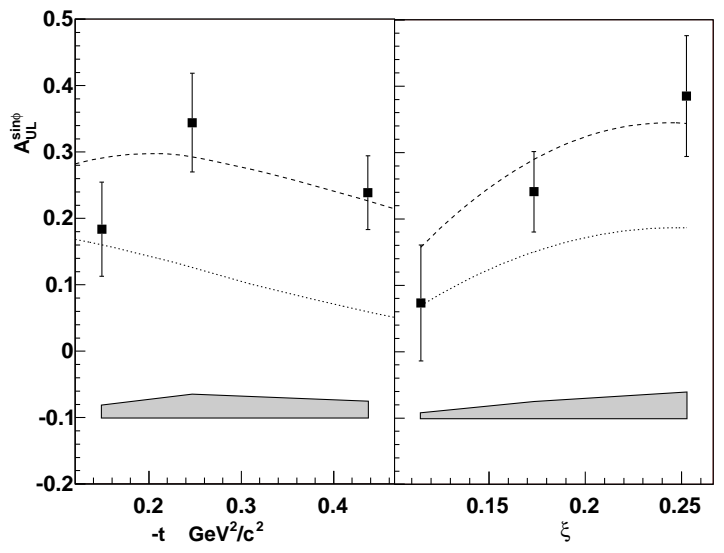

FIGURE 4. The left panel shows the $-t$ dependence of the $\sin \phi$-moment of $A_{\mathrm{UL}}$ for exclusive electroproduction of photons, while the right shows the $\xi$ dependence. The dashed curve is the full model prediction using the $\xi$-dependent GPD parameterization [18] $\left(b_{v a l}=b_{\text {sea }}=1\right.$, and $\left.E=\widetilde{E}=0\right)$. The dotted curve shows the asymmetry when $\widetilde{H}=0$.

previously published CLAS and SAPHIR results. Statistical analysis showed that quoted significance of $5.2 \sigma$ for the peak at $1.54 \mathrm{GeV}$ in low statistics CLAS data [21] was due to underestimate of the background. Upper limits of production cross sections for reactions $\gamma d \rightarrow \Lambda \Theta^{+}, 5 \mathrm{nb}, \gamma n \rightarrow K^{-} \Theta^{+}, 3 \mathrm{nb}, \gamma p \rightarrow \bar{K}^{0} \Theta^{+}, 0.8 \mathrm{nb}$, were extracted $[22,23,24,25]$. Taken together, these new results cast serious doubt on the existence of $\Theta^{+}$pentaquark. An upper limit of $0.2 \mathrm{nb}$ was also obtained for the cross section of $\Theta^{++}$ photoproduction in the reaction $\gamma p \rightarrow \bar{K}^{-} \Theta^{++}[26]$. 


\section{SUMMARY}

A broad physics program, adopted with CLAS, covers topics of nucleon and nuclear structure, formfactors and spin structure functions, hadron spectroscopy and hadronization. A large amount of data have been collected with polarized and unpolarized electron and photon beams in the energy range from $1 \mathrm{GeV}$ to $5.7 \mathrm{GeV}$ using a variety of targets. New detector additions to the base equipment, such as small angle $\mathrm{PbWO}_{4}$ calorimeter and the radial TPC for low energy proton detection, open avenues for a new high precision experiments, not accessible before. Most of the physics program will be continued with the upgraded CEBAF machine, at $11 \mathrm{GeV}$, using the CLAS12 detector.

\section{ACKNOWLEDGMENTS}

This work authored by The Southeastern Universities Research Association, Inc. under U.S. DOE Contract No. DE-AC05-84150. The U.S. Government retains a non-exclusive, paid-up, irrevocable, world-wide license to publish or reproduce this manuscript for U.S. Government purposes.

\section{REFERENCES}

1. B.Mecking et al.,Nucl. Instr. Meth. A 440, 263 (2000).

2. I.G. Aznauryan et al., Phys. Rev. C 71, 015201 (2005).

3. H. Egiyan et al., Phys. Rev. C 73, 025204 (2006).

4. M. Ungaro et al., hep-ex/0606042 (2006).

5. S. Capstick and W. Roberts, Phys. Rev. D 58, 074011 (1998).

6. R. Bradford et al.,Phys. Rev. C 73, 035202 (2006)

7. S.C. Pieper, R.B.Wiringa,V.R. Pandharipande Phys. Rev. C 46, 1741 (1992).

8. C. Ciofi degli Atti, S. Simula, Phys. Rev. C53, 1689 (1996).

9. L.L. Frankfurt and M.I. Strikman, Phys. Rep. 76, 215 (1981).

10. K.Sh. Egiyan et al., Phys. Rev. C 68, 014313 (2003).

11. K.Sh. Egiyan et al., Phys. Rev. Lett. 96, 082501 (2006).

12. K.Sh. Egiyan et al., CLAS-NOTE 2005-004 (2005).

13. A. Nogga et al., Phys. Rev. C 67, 034004 (2003).

14. X. Ji, Phys. Rev. Lett. 78, 610 (1997); Phys. Rev. D 55, 7114 (1997).

15. A.V. Radyushkin, Phys. Lett. B 380, 417 (1996); Phys. Rev. D 56, 5524 (1997).

16. J.C. Collins, L. Franfurt, and M. Strikman, Phys. ReV. D 56, 2982, 1997.

17. S. Stepanyan et al., Phys. Rev. Lett. 87, 182002 (2001).

18. M. Vanderhaeghen, P.A.M. Guichon and M. Guidal, Phys. Rev. D 60, 094017 (1999).

19. F. Cano and J.-M. Laget, Phys.Lett. B551, 317 (2003), Erratum-ibid. B571, 260 (2003), hep$\mathrm{ph} / 0209362$ (2002).

20. Sh.Chen et al., hep-ex/0605012 (2006).

21. S. Stepanyan et al., Phys. Rev. Lett. 91 252001(2003).

22. M. Battaglieri et al., Phys. Rev. Lett. 96, 042001 (2006).

23. S. Niccolai et al., Phys. Rev. Lett. 97, 032001 (2006).

24. B. McKinnon et al., Phys. Rev. Lett 96, 212001 (2006).

25. R. De Vita et al., Phys. Rev. D 74, 032001 (2006).

26. V. Koubarovski et al., hep-ex/0605001 (2006). 